\title{
USE OF SYSTEMIC APPROACH IN RADIATION BIOLOGY*
}

\author{
EwA STĘPIEŃ
}

Department of Medical Physics, The M. Smoluchowski Institute of Physics Faculty of Physics Astronomy and Applied Sciences, Jagiellonian University Łojasiewicza 11, 30-348 Kraków, Poland

\section{FrANCISCO J. ENGUITA}

Instituto de Medicina Molecular, Faculdade de Medicina, Universidade de Lisboa Av. Egas Moniz, 1649-028 Lisbon, Portugal

(Received September 21, 2017)

Various biological systems are characterized with different radiation sensitivity to different damaging factors. The basic idea justified in theoretical and experimental models confirms "the target theory". The principle of a target theory assumes that radiation sensitivity correlates with chemical and morphological characteristics, the size and type of the sensitive structure. The target of enzymes was shown to be the entire molecule, for viruses or bacteria, the total deoxy- or ribonucleic acids (DNA or RNA) strands, in the higher Eukaryotic cells, the specific of arrangements of DNA within chromosomes and epigenetic modifications reinforce the system in the target complexity. In this paper, we would like to introduce the new theoretical and experimental framework for understanding the complexity of biological systems and what we currently know about the genetic and epigenetic mechanisms regulating metabolic and genetic response to radiation on the cellular level, highlighting the differences between a normal and a cancer cell.

DOI:10.5506/APhysPolB.48.1647

\section{Systems biology — where we are and what we know}

When defining systems biology, one must take into account that the intuitive understanding of this specific biology field does not fully cover the

* Presented at the $2^{\text {nd }}$ Jagiellonian Symposium on Fundamental and Applied Subatomic Physics, Kraków, Poland, June 3-11, 2017. 
systemic definition of this biological speciality. Inconsistency in understanding of a definition of systems biology arises from the different understanding of a definition term. In both experiment and theoretical sciences, definitions play an important role in an empirical knowledge-gaining process to understand the development of the ideas in a specific field. Starting from the Aristotelian opinion, there is no agreement what is a genus of systems biology term: biology, as a field of science, of systems, known as complex systems composed of many biological components which interact with each other, as a specific discipline of biology or the systemic approach to understanding of the complexity of biological processes [1].

Ones undertaken definitions can vary, in terms of used terminology, applied methodology, and the role of intuition in the process of understanding or the acceptance of an idea. The time course of systems biology extends from the first application of the general system theory to living organisms proposed by L. von Bertalanffy in his pioneering work in the 1920s, referred in his later publications [2]. In contrast to formerly accepted reductionism and analytical approach (since F. Bacon onwards), von Bertalanffy defined systems biology as a neglecting theory taking into account that the analytical procedure is not adequate to describe the entire system workout, mainly because of assuming a linear nature of interactions between parts and, what is move intuitive, by a separate analysis of individual parts of the system [2]. Starting from this point, the first definition of systems theory was based on the use of engineering approach to description of biological opened-systems.

After better understanding what the genome contains and how it controls an organism (system) on genetic and epigenetic levels, it has to become clear that the human genome does not have much more genes comparing to other species, but this genome is much more complex as we expected [3]. Thus, the systemic approach brings the only opportunity to reveal the genome complexity in health and disease [4].

Taking into consideration all that needs for better understanding of the systems biology, the modern definition should cover a comprehensive quantitative analysis of the manner in which all the components of a biological system interact functionally over time [5]. Intuitively, systems biology is a biology-based interdisciplinary study field that focuses on the systemic analysis of complex interactions in biological systems. It is rather a holistic way of thinking that most of biologists or physiologists have been using for decades instead of formerly used reductionism for better understanding the entirety of a process and for discovering new emergent properties [6]. Therefore, every definition should consider a delicate balance between four opposing aspects of experimental and theoretical science: 
- Discipline: biology versus physics;

- Method: computational versus experiments;

- Scale: genome-wide versus small-scale networks;

- Analysis: deterministic versus probabilistic.

In the extreme environmental conditions, including radiation or other stressing factors (disease), the level of complexity of a system is increasing [6].

\section{Cells and other examples of complex biology systems}

Cells can vary in terms of their both biological and physical properties. Collection of cell images taken by means of scanning electron microscopy shows the diversity in cell shape: stimulated endothelial cells, erythrocytes in health and heart disease or malignant glioblastoma cells after irradiation with different radiation beam and dose (figures 1-6 in [7]). In physiology, the first system approach was applied in 1964 by E. Davie and O.D. Ratnoff, who had proposed their systemic model of a clotting cascade - the waterfall of blood coagulation [8] (figure 8 in [7]). Clotting is a process distinguished as a secondary hemostasis leading to formation of a thrombus to prevent bleeding. In the early 1960s, the clotting cascade has been defined as interplay of XII coagulation factors, the role of factor XIII and tissue factor was not presented in a proposed model. Further investigations lead to the extended knowledge about the coagulation cascade and allowed to propose a more interactive system [9]. Inclusion to the model a fibrinolysis pathway extends the coagulation system with more factors to form a network of the coagulation system [10]. The extended coagulation system by the KEGG database includes coagulation, fibrinolysis and complement pathways, which make in total 79 proteins [11]. This network can be expressed as a node-interaction system with 828 gene associations described in a STRING defined model (figure 11 in [7]) [12]. The same systemic approach to present the radiation essential Eucaryota cell pathways revealed that in the cell cycle, apoptosis and p53 pathways, the number of proteins is correspondingly high $(124,135$ and 69) with respective associations (2637, 2368 and 585) [7].

\section{How can radiation biology benefit from systems biology?}

Radiation acts on biological systems on different temporal levels, giving both direct and indirect effects (figure 17 in [7]). The final biologic stage depends whether the biological action causes reversible or irreversible molecular (genetic, metabolic) alterations. Finally, the biological system gains a new property (mutation) or enters the death pathway (apoptosis). 
If we consider a living cell as a complex system, the understanding of many cellular reactions after radiation exposure will be plausible and reliable. Radiation can hit every single molecule inside a cell and disturb every metabolic process. In the 1960s, a basic idea of radiation sensitivity was formulated. This is a concept of the target theory, which basically states that radiation sensitivity of the biological system increases with the size of its sensitivity targets. The larger molecule is, the more sensitivity increases. This relationship between DNA volume (length) and radiation was found by Kaplan and Moses to be approximately linear with different radiation sensitivity constant, for high organization of DNA in a biological system, the irradiation probability decreases: for one-strand viruses it was approximately 1 , for two-strand viruses 0.1 , haploid bacteria and yeast 0.02 , diploid bacteria and yeast 0.002 [13]. In contradiction to this theory, the probability to hit the bigger diploid nucleus is higher. The nucleus size expressed as a nucleus dissection area is much higher in diploid cells in comparison to haploid ones. Now we know that this irradiation probability decreases, observed as higher resistance in more complex biological systems, due to improved repair processes in higher cells. Another issue is a dose response and radiation molecular nature. There is a significant difference in cell response to photon and hadron irradiation [14].

Among the number of stress signals including irradiation caused DNA damage, the activation of DNA-damage response pathways mediated by p53 is very important. The p53 protein is employed as a transcriptional activator of p53-regulated genes. This results in three major outputs: cell cycle arrest, cellular senescence and apoptosis [15]. Its role can be varied depending on the cancerogenity of a stressing factor and cell predisposition. Systemic approach to the radiation response of normal and cancer cells, based on a biology-based interdisciplinary study, is the only opportunity to reveal the complex response of biological systems in the radiation biology.

\section{Conclusions}

In the radiation biology, the main stress is put on the sensitivity to radiation factors expressed as different biological effects (functional sensitivity). The effects of radiation show the character of disturbances in cell function originating from the DNA damage, with the strong influence on different metabolic and signaling pathways which play an important part in the progress of these disturbances. Systems biology brings the new insight in better understanding of these pathways interactions. 


\section{REFERENCES}

[1] R. Kublikowski, Summarium 45, 45 (2016).

[2] L. von Bertalanffy, General System Theory. Foundations, Development, Applications, George Braziller, Inc., New York 1969.

[3] A. Kundaje [Roadmap Epigenomics Consortium], Nature 518, 317 (2015).

[4] L. Diaz-Beltran et al., Behav. Sci. 3, 253 (2013).

[5] A. Aderem, Cell 121, 511 (2005).

[6] L.M. Edwards, I. Thiele, Extrem Physiol. Med. 2, 8 (2013).

[7] E. Stępień, Presentation: Use of systemic approach in radiation biology, http://koza.if .uj.edu.pl/jagiellonian-symposium-2017/program

[8] E.W. Davie, J. Biol. Chem. 278, 50819 (2003).

[9] S.G. Uzlova, K.G. Guria, G.T. Guria, Philos. Trans. A Math. Phys. Eng. Sci. 366, 3649 (2008).

[10] M.G. Crooks, S.P. Hart, Eur. Respir. Rev. 24, 392 (2015).

[11] http://www.genome.jp/kegg/pathway.html accession date: 05-08-2017.

[12] D. Szklarczyk et al., Nucleic Acids Res. 45, D362 (2017).

[13] H.S. Kaplan, L.E. Moses, Science 145, 21 (1964).

[14] J. Miszczyk et al., Radiother. Oncol. 115, 128 (2015).

[15] D.W. Meek, Biochem. J. 469, 325 (2015). 